Bangladesh J. Sci. Res. 27(1): 87-98, 2014 (June)

\title{
GENERALIZED DERIVATIONS ON LIE IDEALS OF COMPLETELY SEMIPRIME $\Gamma$-RINGS
}

\author{
M. M. Rahman ${ }^{*}$ and A. C. Paul ${ }^{1}$ \\ Department of Mathematics, Jagannath University, Dhaka-1100, Bangladesh.
}

\begin{abstract}
In this paper we prove that under some suitable conditions, every Jordan generalized derivation on Lie ideals of a 2-torsion free completely semiprime $\Gamma$-ring is a generalized derivation on the same.
\end{abstract}

Key words: Generalized derivation, Lie ideal, completely semiprime $\Gamma$-ring

\section{Introduction}

The notion of generalized derivation was introduced by Hvala (1998) and Bresar (1991). Afterwards, many authors have investigated comparable results on prime and semiprime rings with generalized derivtions. The notions of generalized derivation and Jordan generalized derivation of $\Gamma$-rings have been introduced by Ceven and Ozturk (2004) as below.

If $M$ is a $\Gamma$-ring. An additive mapping $f: M \rightarrow M$ is said to be a generalized derivation if there exists an additive derivation $d: M \rightarrow M$ such that $f(a \alpha b)=f(a) \alpha b+a \alpha d(b), \forall a, b \in M$ and $\alpha \in \Gamma$ and $f$ is a Jordan generalized derivation if $f(a \alpha a)=f(a) \alpha a+a \alpha d(a), \forall a \in M$ and $\alpha \in \Gamma$. The concept of a $\Gamma$ ring was first introduced by Nobusawa (1964) and afterwards it was generalized by Barnes (1966). Many properties of $\Gamma$-rings were obtained by Barnes (1966), Kyuno (1978), Luh (1969), Nobusawa (1964) and others.

Let $M$ and $\Gamma$ be additive abelian groups. If there is a mapping $M \times \Gamma \times M \rightarrow M$ such that the conditions

$$
\begin{aligned}
& \cdot(x+y) \alpha z=x \alpha z+y \alpha z, x(\alpha+\beta) y=x \alpha y+x \beta y, x \alpha(y+z)=x \alpha y+x \alpha z \\
& \cdot(x \alpha y) \beta z=x \alpha(y \beta z)
\end{aligned}
$$

are satisfied for all $x, y, z \in M$ and $\alpha, \beta \in \Gamma$ then $M$ is called a $\Gamma$-ring. This concept is more general than a ring. A $\Gamma$-ring $M$ is called semiprime if $a \Gamma M \Gamma a=0$ (with $a \in M$ ) implies $a=0$ and $M$ is called completely semiprime if $a \Gamma a=0$ (with

\footnotetext{
* Corresponding author: <mizanorrahman@gmail.com> ${ }^{1}$ Department of Mathematics, University
} of Rajshahi, Rajshahi-6205, Bangladesh. 
$a \in M$ ) implies $a=0$. A $\Gamma$-ring $M$ is 2-torsion free if $2 a=0$ implies $a=0, \forall a \in M$. For any $x, y \in M$ and $\alpha \in \Gamma$, we denote the commutator $x \alpha y-y \alpha x$ by $[x, y]_{\alpha}$. An additive subgroup $U \subseteq M$ is said to be a Lie ideal of $M$ if whenever $u \in U, m \in M$ and $\alpha \in \Gamma$ then $[u, m]_{\alpha} \in U$. In the main result of this article, we assume that the Lie ideal $U$ satisfies $u \alpha u \in U, \forall u \in U, \alpha \in \Gamma$. A Lie ideal of this type is called a square closed Lie ideal. Furthermore, if the Lie ideal $U$ is square closed and $U \nsubseteq Z(M)$, where $Z(M)$ denotes the centre of $M$ then $U$ is called an admissible Lie ideal of $M$.

In 1984, Awtar (1984) extended a well known result to Lie ideals proved by Herstein (1957) which states that "every Jordan derivation on a 2-torsion free prime ring is a derivation”. He proved that if $U \nsubseteq Z$, is a square closed Lie ideal of a 2-torsion free prime ring $R$ and $d: R \rightarrow R$ is an additive mapping such that $d\left(u^{2}\right)=d(u) u+u d(u), \forall u \in U$ then $d(u v)=d(u) v+u d(v), \forall u, v \in U$.

Ashraf and Rehman (2000) studied on Lie ideals and Jordan left derivations of prime rings. They proved that if $d: R \rightarrow R$ is an additive mapping on a 2-torsion free prime ring $R$ satisfying $d\left(u^{2}\right)=2 u d(u), \forall u \in U$, where $U$ is a Lie ideal of $R$ such that $u^{2} \in U, \forall u \in U$, then $d(u v)=d(u) v+u d(v), \forall u, v \in U$. Halder and Paul (2012) extended the results of Ceven (2002) on Lie ideals.

In the present paper, we assume that $M$ is a completely semiprime $\Gamma$-ring satisfying the condition $\left(^{*}\right) a \alpha b \beta c=a \beta b \alpha c, \forall a, b, c \in M$ and $\alpha, \beta \in \Gamma$ and $U$ be an admissible Lie ideal of $M$. We prove that, if $f: M \rightarrow M$ is a Jordan generalized derivation on $U$ of $M$ with an associated Jordan derivation $d$ on $U$ of $M$ and $f(a) \alpha b=f(b) \alpha a$ and $a \alpha d(b)=b \alpha d(a)$ holds $\forall a, b \in U$ and $\alpha \in \Gamma$ then $f$ is a generalized derivation on $U$ of $M$.

\section{Jordan Generalized Derivations on Lie Ideals of Completely Semiprime $\Gamma$-Rings}

We introduce the concept of Jordan generalized derivation and generalized derivation on Lie ideals of a $\Gamma$-ring in the following way.

Definition 1. Let $M$ be a $\Gamma$-ring and $U$ be a Lie ideal of $M$. An additive mapping $f: M \rightarrow M$ is said to be a Jordan generalized derivation on $U$ if there exists a mapping $d: M \rightarrow M$ on $U$ such that $f(u \alpha u)=f(u) \alpha u+u \alpha d(u), \forall u \in U$ and $\alpha \in \Gamma$ 
and $f: M \rightarrow M$ is said to be a generalized derivation on $U$ if there exists a mapping $d: M \rightarrow M$ on $U$ such that $f(u \alpha v)=f(u) \alpha v+u \alpha d(v)$ for all $u, v \in U$ and $\alpha \in \Gamma$.

Example 1. Not every Jordan generalized derivation on Lie ideal is a generalized derivation. Let $M$ be a $\Gamma$-ring satisfying the condition (*) and let $U$ be a Lie ideal of $M$. Let $a \in M$ and $\alpha \in \Gamma$ be fixed elements. Define $f: M \rightarrow M$ by $f(x)=a \alpha x+x \alpha a$ and $d(x)=x \alpha a-a \alpha x$ for all $x \in U$. Then using the condition (*), for all $y \in U$ and $\beta \in \Gamma$,

$f(y \beta y)=a \alpha y \beta y+y \beta y \alpha a=a \alpha y \beta y+y \alpha a \beta y-y \alpha a \beta y+y \beta y \alpha a=(a \alpha y+y \alpha a) \beta y$

$+y \beta(y \alpha a-a \alpha y)$

$=f(y) \beta y+y \beta d(y)$ for every $y \in U$ and $\beta \in \Gamma$.

Therefore, $f$ is a Jordan generalized derivation on $U$.

Also, for all $x, y \in U$ and $\beta \in \Gamma$, we have

$f(x \beta y)=a \alpha x \beta y+x \beta y \alpha a=a \alpha x \beta y+x \alpha a \beta y-x \alpha a \beta y+x \beta y \alpha a=(a \alpha x+x \alpha a) \beta y$

$+x \beta(y \alpha a-a \alpha y)$

$=f(x) \beta y+x \beta d(y)$, for every $x, y \in U$ and $\beta \in \Gamma$.

Therefore, $f$ is a generalized derivation on $U$.

Now, let $f: M \rightarrow M$ be a generalized derivation with an associated derivation $d$ on $U$ Let $M_{1}=\{(x, x): x \in M\}$ and $\Gamma_{1}=\{(\alpha, \alpha): \alpha \in \Gamma\}$.

If we define addition and multiplication on $M_{1}$ by $(x, x)+(y, y)=(x+y, x+y)$ and $(x, x)(\alpha, \alpha)(y, y)=(x \alpha y, x \alpha y)$, then $M_{1}$ is a $\Gamma_{1}$-ring.

If we define $U_{1}=\{(u, u): u \in U\}$, then for $u \alpha x-x \alpha u \in U$

$$
\begin{aligned}
(u, u)(\alpha, \alpha)(x, x)-(x, x)(\alpha, \alpha)(u, u) & =(u \alpha x, u \alpha x)-(x \alpha u, x \alpha u) \\
& =(u \alpha x-x \alpha u, u \alpha x-x \alpha u) \in U_{1}
\end{aligned}
$$

Hence, $U_{1}$ is a Lie ideal of $M_{1}$.

Now, we define a mapping $F: M_{1} \rightarrow M_{1}$ by $F((u, u))=(f(u), f(u))$ and $D((u, u))=(d(u), d(u))$. 
Then it is clear that $F$ is a Jordan generalized derivation on $U$ with an associated derivation $D$ on $U$ which is not a generalized derivation on $U$.

In order to prove our desired result we establish a sequence of lemmas as follows.

Lemma 1. Let $M$ be a $\Gamma$-ring and $U$ be a Lie ideal of $M$ such that $u \alpha u \in U, \forall u \in U$ and $\alpha \in \Gamma$. If $f$ is a Jordan generalized derivation with an associated Jordan derivation $d$ on $U$ of $M$ then $\forall a, b, c \in U$ and $\alpha, \beta \in \Gamma$, the following statements hold.

(i) $f(a \alpha b+b \alpha a)=f(a) \alpha b+f(b) \alpha a+a \alpha d(b)+b \alpha d(a)$.

(ii) $f(a \alpha b \beta a+a \beta b \alpha a)=f(a) \alpha b \beta a+f(a) \beta b \alpha a+a \alpha d(b) \beta a+a \beta d(b) \alpha a$

$+a \alpha b \beta d(a)+a \beta b \beta d(a)$

In particular, if $M$ is 2-torsion free and satisfies the condition (*), then

(iii) $f(a \alpha b \beta a)=f(a) \alpha b \beta a+a \alpha d(b) \beta a+a \alpha b \beta d(a)$.

(iv) $f(a \alpha b \beta c+c \alpha b \beta a)=f(a) \alpha b \beta c+f(c) \alpha b \beta a+a \alpha d(b) \beta c+c \alpha d(b) \beta a+$ $a \alpha b \beta d(c)+c \alpha b \beta d(a)$.

Proof. Since $U$ is a Lie ideal satisfying the condition $a \alpha a \in U, \forall a \in U, \alpha \in \Gamma$.

For $a, b \in U, \alpha \in \Gamma,(a \alpha b+b \alpha a)=(a+b) \alpha(a+b)-(a \alpha a+b \alpha b)$ and so $(a \alpha b+b \alpha a) \in U$.

Also, $[a, b]_{\alpha}=a \alpha b-b \alpha a \in U$ and it follows that $2 a \alpha b \in U$.

Hence $4 a \alpha b \beta c=2(2 a \alpha b) \beta c \in U, \forall a, b, c \in U, \alpha, \beta \in \Gamma$. Thus,

$$
\begin{aligned}
f(a \alpha b+b \alpha a)= & f((a+b) \alpha(a+b)-(a \alpha a+b \alpha b)) \\
= & f(a+b) \alpha(a+b)+(a+b) \alpha d(a+b)-f(a) \alpha a-a \alpha d(a) \\
& -f(b) \alpha b-b \alpha d(b) \\
& =f(a) \alpha a+f(a) \alpha b+f(b) \alpha a+f(b) \alpha b+a \alpha d(a)+a \alpha d(b)+b \alpha d(a) \\
& +b \alpha d(b)-f(a) \alpha a-a \alpha d(a)-f(b) \alpha b-b \alpha d(b) \\
& =f(a) \alpha b+a \alpha d(b)+f(b) \alpha a+b \alpha d(a) .
\end{aligned}
$$

Replacing $b$ by $a \beta b+b \beta a$ in (i), we get

$$
\begin{aligned}
& f(a \alpha(a \beta b+b \beta a)+(a \beta b+b \beta a) \alpha a) \\
& =f(a) \alpha(a \beta b+b \beta a)+a \alpha d(a \beta b+b \beta a)+f(a \beta b+b \beta a) \alpha a+(a \beta b+b \beta a) \alpha d(a) .
\end{aligned}
$$

This implies, 
$f(a \alpha a) \beta b+(a \alpha a) \beta d(b)+f(b) \beta(a \alpha a)+b \beta d(a \alpha a)+f(a \alpha b \beta a+a \beta b \alpha a)$

$=f(a) \alpha a \beta b+f(a) \alpha b \beta a+a \alpha d(a) \beta b+a \alpha a \beta d(b)+a \alpha d(b) \beta a+a \alpha b \beta d(a)$

$+f(a) \beta b \alpha a+a \beta d(b) \alpha a+f(b) \beta a \alpha a+b \beta d(a) \alpha a+a \beta b \alpha d(a)+b \beta a \alpha d(a)$.

This implies,

$f(a) \alpha a \beta b+a \alpha d(a) \beta b+a \alpha a \beta d(b)+f(b) \beta a \alpha a+b \beta d(a) \alpha a+b \beta a \alpha d(a)$

$+f(a \alpha b \beta a+a \beta b \alpha a)$

$=f(a) \alpha a \beta b+f(a) \alpha b \beta a+a \alpha d(a) \beta b+a \alpha a \beta d(b)+a \alpha d(b) \beta a+a \alpha b \beta d(a)+f(a) \beta b \alpha a$

$+a \beta d(b) \alpha a+f(b) \beta a \alpha a+b \beta d(a) \alpha a+a \beta b \alpha d(a)+b \beta a \alpha d(a)$.

Now, cancelling the like terms from both sides we get the required result. Using the condition (*) and since $M$ is 2-torsion free, (iii) follows from (ii). And finally (iv) is obtained by replacing $a$ by $a+c$ in (iii).

Definition 2. Let $M$ be a 2-torsion free semiprime $\Gamma$-ring satisfying the condition $\left(^{*}\right)$ and $U$ be a Lie ideal of $M$. Let $f$ be a Jordan generalized derivation with an associated Jordan derivationd on $U$ of $M$. Then $\forall a, b \in U$ and $\alpha \in \Gamma$, we define $G_{\alpha}(a, b)=f(a \alpha b)-f(a) \alpha b-a \alpha d(b)$.

Remark 1. $f$ is a generalized derivation on $U$ of $M$ if and only if $G_{\alpha}(a, b)=0, \forall a, b \in U$ and $\alpha \in \Gamma$.

Lemma 2. Let $M$ be 2-torsion free semiprime $\Gamma$-ring satisfying the condition (*) and $U$ be a square closed Lie ideal of $M$. Let $f$ be a Jordan generalized derivation with an associated Jordan derivation $d$ on $U$ of $M$. Then $\forall a, b, c \in U$ and $\alpha, \beta \in \Gamma$, the following statements hold:

(i) $G_{\alpha}(a, b)+G_{\alpha}(b, a)=0$; (ii) $G_{\alpha}(a+b, c)=G_{\alpha}(a, c)+G_{\alpha}(b, c)$;

(iii) $G_{\alpha}(a, b+c)=G_{\alpha}(a, b)+G_{\alpha}(a, c)$; (iv) $G_{\alpha+\beta}(a, b)=G_{\alpha}(a, b)+G_{\beta}(a, b)$.

Lemma 3. Let $M$ be a 2-torsion free completely semiprime $\Gamma$-ring satisfying the condition (*) and $U$ be a Lie ideal of $M$. If $u \in U$ such that $\left[u,[u, x]_{\alpha}\right]_{\alpha}=0, \forall x \in M$ and $\alpha \in \Gamma$, then $[u, x]_{\alpha}=0$.

Proof. We have $\left[u,[u, x]_{\alpha}\right]_{\alpha}=0, \forall x \in M$ and $\alpha \in \Gamma$. For every $\beta \in \Gamma$, replacing $x$ by $x \beta x$, we obtain 


$$
\begin{aligned}
0 & =\left[u,[u, x \beta x]_{\alpha}\right]_{\alpha} \\
& =\left[u, x \beta[u, x]_{\alpha}+[u, x]_{\alpha} \beta x\right]_{\alpha} \\
& =\left[u, x \beta[u, x]_{\alpha}\right]_{\alpha}+\left[u,[u, x]_{\alpha} \beta x\right]_{\alpha} \\
& =x \beta\left[u,[u, x]_{\alpha}\right]_{\alpha}+[u, x]_{\alpha} \beta[u, x]_{\alpha}+\left[u,[u, x]_{\alpha}\right]_{\alpha} \beta x+[u, x]_{\alpha} \beta[u, x]_{\alpha} \\
& =2[u, x]_{\alpha} \beta[u, x]_{\alpha} .
\end{aligned}
$$

By the 2-torsion freeness of $M$, we obtain $[u, x]_{\alpha} \beta[u, x]_{\alpha}=0$. Since $M$ is completely semiprime $\Gamma$-ring, hence $[u, x]_{\alpha}=0, \forall x \in M$ and $\alpha \in \Gamma$.

Lemma 4. Let $M$ be a 2-torsion free completely semiprime $\Gamma$-ring satisfying the condition (*) and $U$ be a commutative Lie ideal of $M$, then $U \subseteq Z(M)$.

Proof. Since $U$ is a commutative Lie ideal of $M$, so we have $\left[u,[u, x]_{\alpha}\right]_{\alpha}=0, \forall u \in U, x \in M$ and $\alpha \in \Gamma$. Then by Lemma 3, we get $[u, x]_{\alpha}=0$. This implies that $U \subseteq Z(M)$.

Lemma 5. Let $M$ be a 2-torsion free completely semiprime $\Gamma$-ring satisfying the condition (*). If $U \neq 0$ is a sub- $\Gamma$-ring and a Lie ideal of $M$, then either $U \subseteq Z(M)$ or $U$ contains a non-zero ideal of $M$.

Proof. If $U$ is commutative, then by Lemma $4, U \subseteq Z(M)$. So, let $U$ be noncommutative, then for some $u, v \in M$ and $\alpha \in \Gamma$, we have $[u, v]_{\alpha} \in U$. Hence there exists an ideal $J$ of $M$ generated by $[u, v]_{\alpha} \neq 0$ and $J \subseteq U$.

Lemma 6. Let $M$ be a 2-torsion free completely semiprime $\Gamma$-ring satisfying the

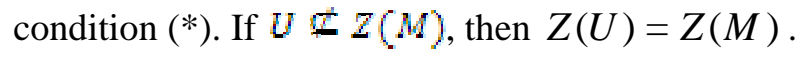

Proof. $Z(U)$ is both a sub- $\Gamma$-ring and a Lie ideal of $M$ such that $Z(U)$ does not contain non-zero ideal of $M$. Therefore in view of Lemma 5, we obtain that $Z(U) \subseteq Z(M)$. Hence, $Z(U)=Z(M)$.

Lemma 7. Let $M$ be a 2-torsion free completely semiprime $\Gamma$-ring satisfying the condition $\left(^{*}\right)$ and $U$ be a Lie ideal of $M$, then $Z\left([U, U]_{\Gamma}\right)=Z(U)$.

Proof. Let $a \in M$ be any element. If $\left[a,[U, U]_{\Gamma}\right]_{\Gamma}=0$, then we prove that $[a, U]_{\Gamma}=0$. This yields that $Z\left([U, U]_{\Gamma}\right)=Z(U)$. If $[U, U]_{\Gamma} \not \subset Z(M)$, then by Lemma 6, $a \in Z(U)$. So $a$ centralize $U$. 
On the other hand, let $[U, U]_{\Gamma} \subseteq Z(M)$. Then we have $\left[u,[u, a]_{\alpha}\right]_{\alpha}=0, \forall u \in U, a \in M$ and $\alpha \in \Gamma$. Thus in view of Lemma 4, we obtain that $[u, a]_{\alpha}=0, \forall u \in U, a \in M$ and $\alpha \in \Gamma$. This gives that $a \in Z(U)$. Hence we have the required result.

Lemma 8. Let $M$ be 2-torsion free completely semiprime $\Gamma$-ring satisfying the condition (*) and $U$ be a Lie ideal of $M$. Let $f$ be a Jordan generalized derivation with an associated Jordan derivation $d$ on $U$ of $M$. Then $G_{\alpha}(a, b) \beta[a, b]_{\alpha}+[a, b]_{\alpha} \beta G_{\alpha}(a, b)=0, \forall a, b \in U$ and $\alpha, \beta \in \Gamma$.

Proof. For any $a, b \in U$ and $\alpha, \beta \in \Gamma$, let $w=4(a \alpha b \beta b \alpha a+b \alpha a \beta a \alpha b)$. Then, using Lemma 1(i)

$$
\begin{aligned}
f(w) & =f((2 a \alpha b) \beta(2 b \alpha a)+(2 b \alpha a) \beta(2 a \alpha b)) \\
& =4 f(a \alpha b) \beta(b \alpha a)+4(a \alpha b) \beta d(b \alpha a)+4 f(b \alpha a) \beta(a \alpha b)+4(b \alpha a) \beta d(a \alpha b) .
\end{aligned}
$$

On the other hand, using Lemma 1(iii)

$$
\begin{aligned}
f(w) & =f(2(a \alpha(2 b \beta b) \alpha a)+2(b \alpha(2 a \beta a) \alpha b)) \\
& =2 f(a) \alpha(2 b \beta b) \alpha a+2 a \alpha d(2 b \beta b) \alpha a+2 a \alpha(2 b \beta b) \alpha d(a)+2 f(b) \alpha(2 a \beta a) \alpha b \\
& +2 b \alpha d(2 a \beta a) \alpha b+2 b \alpha(2 a \beta a) \alpha d(b) \\
& =4 f(a) \alpha b \beta b \alpha a+4 a \alpha d(b) \beta b \alpha a+4 a \alpha b \beta d(b) \alpha a+4 a \alpha b \beta b \alpha d(a) \\
& +4 f(b) \alpha a \beta a \alpha b+4 b \alpha d(a) \beta a \alpha b+4 b \alpha a \beta d(a) \alpha b+4 b \alpha a \beta a \alpha d(b) .
\end{aligned}
$$

Equating the two expressions for $f(w)$, we get

$4(f(a \alpha b)-f(a) \alpha b-a \alpha d(b)) \beta b \alpha a+4(f(b \alpha a)-f(b) \alpha a-b \alpha d(a)) \beta a \alpha b+$ $4 a \alpha b \beta(d(b \alpha a)-f(b) \alpha a-b \alpha d(a))+b \alpha a \beta(d(a \alpha b)-f(a) \alpha b-a \alpha d(b))=0$.

Now, using the definition 2, we obtain

$4 G_{\alpha}(a, b) \beta b \alpha a+4 G_{\alpha}(b, a) \beta a \alpha b+4 a \alpha b \beta G_{\alpha}(b, a)+4 b \alpha a \beta G_{\alpha}(a, b)=0$.

Using Lemma 2(i), we have

$4 G_{\alpha}(a, b) \beta b \alpha a-4 G_{\alpha}(a, b) \beta a \alpha b-4 a \alpha b \beta G_{\alpha}(a, b)+4 b \alpha a \beta G_{\alpha}(a, b)=0$.

By the 2-torsion freeness of $M$, we get

$G_{\alpha}(a, b) \beta[a, b]_{\alpha}+[a, b]_{\alpha} \beta G_{\alpha}(a, b)=0, \forall a, b \in U$ and $\alpha, \beta \in \Gamma$. 
Lemma 9. Let $M$ be a 2-torsion free completely semiprime $\Gamma$-ring, $U$ be a Lie ideal of $M$ and let $a, b \in U$ and $\alpha \in \Gamma$. If $a \alpha b+b \alpha a=0$ then $a \alpha b=0=b \alpha a$.

Proof. Let $\delta \in \Gamma$ be any element. Suppose that $a, b \in U$ and $\alpha \in \Gamma$ such that $a \alpha b+b \alpha a=0$. Using the relation $a \alpha b=-b \alpha a$ repeatedly, we get

$$
\begin{aligned}
4(a \alpha b) \delta(a \alpha b) & =-4(b \alpha a) \delta(a \alpha b)=-4(b(\alpha a \delta) a) \alpha b \\
& =4(a(\alpha a \delta) b) \alpha b=2 a \alpha(2 a \delta b) \alpha b \\
& =-2 a \alpha(2 b \delta a) \alpha b=-4(a \alpha b) \delta(a \alpha b) .
\end{aligned}
$$

This implies,

$$
8((a \alpha b) \delta(a \alpha b))=0 .
$$

Since $M$ is 2-torsion free,

$$
(a \alpha b) \delta(a \alpha b)=0
$$

Therefore,

$$
(a \alpha b) \Gamma(a \alpha b)=0 .
$$

By the complete semiprimeness of $M$, we get $a \alpha b=0$.

Similarly, it can be shown that, $b \alpha a=0$.

Corollary 1. Let $M$ be a 2-torsion free completely semiprime $\Gamma$-ring satisfying the condition (*), $U$ be a Lie ideal of $M$ and $f$ be a Jordan generalized derivation with an associated Jordan derivation $d$ on $x$ of $M$. Then $\forall a, b \in U$ and $\alpha, \beta \in \Gamma$ (i) $G_{\alpha}(a, b) \beta[a, b]_{\alpha}=0 ;(i i)[a, b]_{\alpha} \beta G_{\alpha}(a, b)=0$.

Proof. Applying the result of Lemma 9 in that of Lemma 8, we obtain these results.

Lemma 10. Let $M$ be a 2-torsion free completely semiprime $\Gamma$-ring satisfying the condition (*), $U$ be a Lie ideal of $M$ and let $f$ be a Jordan generalized derivation with an associated Jordan derivation $d$ on $U$ of $M$. Then $\forall a, b, x, y \in U$ and $\alpha, \beta, \gamma \in \Gamma$ :

(i) $\quad G_{\alpha}(a, b) \beta[x, y]_{\alpha}=0 ; \quad$ (ii) $\quad[x, y]_{\alpha} \beta G_{\alpha}(a, b)=0$

(iii) $G_{\alpha}(a, b) \beta[x, y]_{\gamma}=0$; (iv) $[x, y]_{\gamma} \beta G_{\alpha}(a, b)=0$. 
Proof. (i) If we substitute $a+x$ for $a$ in the Corollary 1(i), then we get

$$
G_{\alpha}(a+x, b) \beta[a+x, b]_{\alpha}=0
$$

Using Lemma 2(ii), we have

$$
G_{\alpha}(a, b) \beta[a, b]_{\alpha}+G_{\alpha}(a, b) \beta[x, b]_{\alpha}+G_{\alpha}(x, b) \beta[a, b]_{\alpha}+G_{\alpha}(x, b) \beta[x, b]_{\alpha}=0 .
$$

Now, using Corollary 1(i), we obtain

$$
G_{\alpha}(a, b) \beta[x, b]_{\alpha}+G_{\alpha}(x, b) \beta[a, b]_{\alpha}=0 .
$$

That is $G_{\alpha}(a, b) \beta[x, b]_{\alpha}=-G_{\alpha}(x, b) \beta[a, b]_{\alpha}$.

Now

$\left(G_{\alpha}(a, b) \beta[x, b]_{\alpha}\right) \beta\left(G_{\alpha}(a, b) \beta[x, b]_{\alpha}\right)=-G_{\alpha}(a, b) \beta[x, b]_{\alpha} \beta G_{\alpha}(x, b) \beta[a, b]_{\alpha}=0$.

Hence, by the complete semiprimeness of $M$, we obtain

$$
G_{\alpha}(a, b) \beta[x, b]_{\alpha}=0 .
$$

Similarly, by replacing $b+y$ for $b$ in this result, we get

$$
G_{\alpha}(a, b) \beta[x, y]_{\alpha}=0 .
$$

(ii) Proceeding in the same way as described above by the similar replacements successively in Corollary 1(ii), we obtain

$$
[x, y]_{\alpha} \beta G_{\alpha}(a, b)=0, \forall a, b, x, y \in M \text { and } \alpha, \beta \in \Gamma \text {. }
$$

(iii) Replacing $\alpha+\gamma$ for $\alpha$ in (i), we get

$$
G_{\alpha+\gamma}(a, b) \beta[x, y]_{\alpha+\gamma}=0 .
$$

By using Lemma 2(iv), we have

$$
\left(G_{\alpha}(a, b)+G_{\gamma}(a, b)\right) \beta\left([x, y]_{\alpha}+[x, y]_{\gamma}\right)=0 .
$$

This implies,

$$
G_{\alpha}(a, b) \beta[x, y]_{\alpha}+G_{\alpha}(a, b) \beta[x, y]_{\gamma}+G_{\gamma}(a, b) \beta[x, y]_{\alpha}+G_{\gamma}(a, b) \beta[x, y]_{\gamma}=0 .
$$

Thus, using (i), we get

$$
G_{\alpha}(a, b) \beta[x, y]_{\gamma}+G_{\gamma}(a, b) \beta[x, y]_{\alpha}=0 .
$$


That is, $G_{\alpha}(a, b) \beta[x, y]_{\gamma}=-G_{\gamma}(a, b) \beta[x, y]_{\alpha}$.

Thus, we have

$\left(G_{\alpha}(a, b) \beta[x, y]_{\gamma}\right) \beta\left(G_{\alpha}(a, b) \beta[x, y]_{\gamma}\right)=-G_{\alpha}(a, b) \beta[x, y]_{\gamma} \beta G_{\gamma}(a, b) \beta[x, y]_{\alpha}=0$.

Hence, by the complete semiprimeness of $M$, we obtain

$$
G_{\alpha}(a, b) \beta[x, y]_{\gamma}=0 .
$$

(iv) By performing the similar replacement in (ii) (as in the proof of (iii)), we get this result.

Remark 2. If $U$ is a commutative Lie ideal of $M$, then $U \subseteq Z(M)$. So by Lemma 1(i) and using 2-torsion freeness of $M$, we get $f(a \alpha b)=f(a) \alpha b+a \alpha d(b), \forall a, b \in U$ and $\alpha \in \Gamma$. Thus for the next results, we assume that $U \leftarrow Z(M)$.

Theorem 1. Let $M$ be a 2-torsion free completely semiprime $\Gamma$-ring satisfying the condition (*), $U$ be an admissible Lie ideal of $M$ and $f$ be a Jordan generalized derivation with an associated Jordan derivation $d$ on $U$ of $M$. If $f(a) \alpha b=f(b) \alpha a$ and $a \alpha d(b)=b \alpha d(a)$ holds $\forall a, b \in U$ and $\alpha \in \Gamma$, then $f$ is a generalized derivation on $U$ of $M$.

Proof. By Lemma 10(iii), we have $G_{\alpha}(a, b) \beta[x, y]_{\gamma}=0, \forall a, b, x, y \in U$ and $\alpha, \beta, \gamma \in \Gamma$.

Also, by Lemma 10(iv), $[x, y]_{\gamma} \beta G_{\alpha}(a, b)=0, \forall a, b, x, y \in U$ and $\alpha, \beta, \gamma \in \Gamma$.

Now, $\left[G_{\alpha}(a, b),[x, y]_{\gamma}\right]_{\beta}=G_{\alpha}(a, b) \beta[x, y]_{\gamma}-[x, y]_{\gamma} \beta G_{\alpha}(a, b)=0$.

Thus, $G_{\alpha}(a, b) \subseteq Z\left([U, U]_{\Gamma}\right)=Z(U)=Z(M)$, by Lemma 6 and Lemma 7 .

Therefore, $G_{\alpha}(a, b) \in Z(M)$. 
Next, we obtain

$$
\begin{aligned}
2 G_{\alpha}(a, b) \beta G_{\alpha}(a, b) & =G_{\alpha}(a, b) \beta\left(G_{\alpha}(a, b)+G_{\alpha}(a, b)\right) \\
& =G_{\alpha}(a, b) \beta\left(G_{\alpha}(a, b)-G_{\alpha}(b, a)\right) \\
& =G_{\alpha}(a, b) \beta(f(a \alpha b)-f(a) \alpha b-a \alpha d(b)-f(b \alpha a)+f(b) \alpha a \\
& +b \alpha d(a)) \\
& =G_{\alpha}(a, b) \beta(f(a \alpha b-b \alpha a)+(b \alpha d(a)-f(a) \alpha b)+(f(b) \alpha a \\
& -a \alpha d(b))) \\
& =G_{\alpha}(a, b) \beta f\left([a, b]_{\alpha}\right) .
\end{aligned}
$$

Therefore, we get

$$
2 G_{\alpha}(a, b) \beta G_{\alpha}(a, b)=G_{\alpha}(a, b) \beta f\left([a, b]_{\alpha}\right) .
$$

Now, by Lemmas 1(i), 10(iii) and 10(iv) with the hypothesis, we obtain

$$
\begin{aligned}
0 & =f\left(G_{\alpha}(a, b) \beta[x, y]_{\gamma}+[x, y]_{\gamma} \beta G_{\alpha}(a, b)\right) \\
& =f\left(G_{\alpha}(a, b)\right) \beta[x, y]_{\gamma}+G_{\alpha}(a, b) \beta d\left([x, y]_{\gamma}\right)+f\left([x, y]_{\gamma}\right) \beta G_{\alpha}(a, b)+[x, y]_{\gamma} \\
& \beta d\left(G_{\alpha}(a, b)\right) \\
& =2 f\left([x, y]_{\gamma}\right) \beta G_{\alpha}(a, b)+2[x, y]_{\gamma} \beta d\left(G_{\alpha}(a, b)\right) .
\end{aligned}
$$

Since $M$ is 2-torsion free, so $f\left([x, y]_{\gamma}\right) \beta G_{\alpha}(a, b)+[x, y]_{\gamma} \beta d\left(G_{\alpha}(a, b)\right)=0$.

That is,

$$
f\left([x, y]_{\gamma}\right) \beta G_{\alpha}(a, b)=-[x, y]_{\gamma} \beta d\left(G_{\alpha}(a, b)\right) .
$$

Then from (1) and (2), we obtain

$$
\begin{aligned}
2 G_{\alpha}(a, b) \beta G_{\alpha}(a, b) \beta G_{\alpha}(a, b) & =G_{\alpha}(a, b) \beta f\left([a, b]_{\alpha}\right) \beta G_{\alpha}(a, b) \\
& =-G_{\alpha}(a, b) \beta[a, b]_{\alpha} \beta d\left(G_{\alpha}(a, b)\right) \\
& =0 .
\end{aligned}
$$

That is, $2 G_{\alpha}(a, b) \beta G_{\alpha}(a, b) \beta G_{\alpha}(a, b)=0$. Since $M$ is 2-torsion free, so we have

$$
G_{\alpha}(a, b) \beta G_{\alpha}(a, b) \beta G_{\alpha}(a, b)=0 .
$$

This shows that, $G_{\alpha}(a, b)$ is a nilpotent element of the completely semiprime $\Gamma$ ring $M$, where $G_{\alpha}(a, b) \in Z(M)$. Since the centre of a completely semiprime $\Gamma$-ring 
does not contain any nonzero nilpotent element, so we get $G_{\alpha}(a, b)=0, \forall a, b \in M$ and $\alpha \in \Gamma$.

\section{References}

Ashraf, M. and N. Rehman. 2000. On Lie ideals and Jordan left derivations of prime rings, Arch. Math. (Brno) 36: 201-206.

Awtar, R. 1984. Lie ideals and Jordan derivations of prime rings. Amer. Math. Soc. 90(1): 9-14.

Barnes, W. E. 1966. On the $\Gamma$-rings of Nobusawa. Pacific J. Math. 18: 411-422.

Bresar, M. 1991. On the distance of the composition of two derivations to the generalized derivations , Glasgow Math . 33: 89-93.

Ceven, Y. 2002. Jordan left derivations on completely prime gamma rings, C. U. Fen-Edebiyat Fakultesi Fen Bilimleri Dergisi 23: 39-43.

Ceven, Y. and M. A. Ozturk. 2004. On Jordan generalized derivations in $\Gamma$-rings, Hacettepe $J$. Math. and Stat 33: 11-14.

Halder, A.K. and A. C. Paul. 2012. Jordan left derivations on Lie ideals of prime $\Gamma$-rings, Punjab University J.Math. 44: 23-29.

Herstein, I.N. 1957. Jordan derivations of prime rings. Proc. Amer. Math. Soc. 8: 1104-1110.

Hvala, B. 1998. Generalized derivations in rings. Comm. Algebra. 26(4): 1147-1166.

Kyuno, S. 1978. On prime gamma rings. Pacific J. Math. 75: 185-190.

Luh , J. 1969. On the theory of simple gamma-rings. Michigan Math. J., 16: 65-75.

Nobusawa, N. 1964. On the generalizeation of the ring theory, Osaka J. Math. 1: 81-89.

(Manuscript received on 14 May, 2014; revised on 14 July, 2014) 